\title{
Knowledge of Human Papillomavirus and its Association with Head and Neck Benign and Malignant Lesions in a Group of Dental Patients in Pakistan
}

\author{
Abdul Samad Gichki', Waranun Buajeeb ${ }^{2}$, Sombhun Doungudomdacha ${ }^{3}$, \\ Siribang-on Pibooniyom Khovidhunkit ${ }^{4 *}$
}

\begin{abstract}
Human papillomaviruses (HPVs) remain a serious world health problem due to their association with cervical and head and neck cancers. While over 100 HPV types have been identified, only a few subtypes are associated with malignancies. HPV 16 and 18 are the most prevalent oncogenic types in head and neck cancers. Although it has been proven that some subsets of benign and malignant head and neck lesions are associated with HPV, the general population have very little awareness and knowledge of their association with HPV. Therefore, the purpose of this study was to determine the knowledge of HPV and its links with head and neck benign and malignant lesions in a group of Pakistani dental patients who attended the Dental Department of the Sandeman provincial hospital in Quetta, Pakistan. One hundred and ninety-two patients were recruited and requested to answer a questionnaire. It was revealed that there was a low level of knowledge about HPV and its association with head and neck benign and malignant lesions among the participants. This result suggested that more education regarding the relationship of HPV in inducing head and neck benign and malignant lesions is required in this group of patients.
\end{abstract}

Keywords: Human papilllomavirus - head and neck cancer - knowledge - Pakistani dental patients

Asian Pac J Cancer Prev, 16 (4), 1581-1585

\section{Introduction}

Human papillomaviruses (HPVs) are small DNA tumor viruses associated with a variety of proliferative diseases. More than 100 types have been identified and can be grouped broadly into cutaneous and mucosal types according to their site of infection and can be subdivided further into low-risk (LR) and high-risk (HR) types depending upon their association with malignancy (Chang et al., 1991). Young age is associated with a high rate of HPV infection with the peak age being less than 25 years old and then declined sharply and HPV infection is more common among males rather than females (Kulasingam et al., 2002; Reiter et al., 2010). HPV can be transmitted sexually through skin to skin contact (Carr and Gyorfi, 2000). It is also reported that HPV can be transmitted from infected mother to baby (Carr and Gyorfi, 2000). In addition, several studies proved that HPV can cause common oral warts, oral papillomas and precancerous and cancerous lesions of the head and neck regions (Prabhu and Wilson, 2013). In the oral cavity, 24 types (HPVs 1, 2, $3,4,6,7,10,11,13,16,18,30,31,32,33,35,45,52,55$, $57,59,69,72$ and 73 ) have been shown to be associated with benign lesions while 12 types (HPVs 2, 3, 6, 11, 13, $16,18,31,33,35,52$ and 57) were related to malignant lesions (Carr and Gyorfi, 2000). It has been reported that HPV 16 is the most identified HPV in oral squamous cell carcinomas (OSCCs) and that HPVs 16 and 18 played important roles in the malignant transformation in OSCCs (Kojima et al., 2003).

Awareness and knowledge of HPV and its association with cervical cancer has been reported in many studies (Waller et al., 2003; Gerend and Magloire, 2008; Kobetz et al., 2010; Montgomery and Smith-Glasgow, 2012). Since the advent of HPV vaccines, a number of studies were conducted to investigate the knowledge of participants about HPV and its association with cervical cancer as well as the interest in receiving HPV vaccine (Saha et al., 2010; Wierzbicka et al., 2014). The percentage of the participants who reported that they have heard of HPV varied between $30-78 \%$ depending on the ethnicity, levels of education, age, experiences in sexual intercourse and admission or attendance in a well woman clinic (Waller et al., 2003; Gerend and Magloire, 2008; Kobetz et al., 2010; Montgomery and Smith-Glasgow, 2012). In spite of the high percentage of the patients who have heard of HPV,

${ }^{1}$ Department of Oral Medicine, Bolan Medical College, Quetta, Pakistan, ${ }^{2}$ Department of Oral Medicine and Periodontology, ${ }^{4}$ Department of Advanced General Dentistry, Faculty of Dentistry, Mahidol University, Bangkok, ${ }^{3}$ Department of Conservative Dentistry, Faculty of Dentistry, Western University, Pathumthani, Thailand *For correspondence: siribangon.pib@mahidol.ac.th 
the general knowledge about HPV was poor and there were a lot of misconceptions and misunderstandings about the characteristics; mode of transmission; contribution of the virus in causing cervical, genital and anal cancers; primary prevention of the virus; and role of HPV vaccine in preventing cervical cancer (Waller et al., 2003; Gerend and Magloire, 2008; Kobetz et al., 2010; Montgomery and Smith-Glasgow, 2012).

Although there were several reports about the knowledge of HPV in association with cervical cancer and cancer of the genital areas, there is no study regarding the knowledge of HPV and its association with head and neck precancerous and cancerous lesions in Pakistan. Given this, the objective of this research was to evaluate the knowledge of dental patients regarding HPV characteristics and its association with head and neck benign and malignant lesions. The result of this study could lead to the promotion of a health care program that will educate patients about the role of HPV in causing head and neck benign and malignant lesions.

\section{Materials and Methods}

\section{Ethical consideration}

The study protocol was reviewed and approved by the committee on human rights in human experimentation, Mahidol university, Thailand (MU-IRB 2010/115.2004). Collection of data regarding the knowledge of participants was performed under the permission and supervision of the head of the Dental Department of the Sandeman Provincial Hospital in Quetta, Balochistan, Pakistan. All details about the volunteers and their identity were anonymized. Each participant was given both verbal and written information about the nature of the study and a written informed consent was required for participation. All individuals were allowed to withdraw from the study at any time during the research.

\section{Study population}

Between May 2010 to June 2010, 192 patients attending the Dental Department of the Sandeman provincial hospital were recruited. The patients were the same as in our previous study (Gichki et al., 2012). The participants consisted of males and females with age between 18-60 years old. All patients who agreed to participate in this study signed an informed consent form. The histories of the patients were collected and documented in the hospital chart. The participants were asked to answer the questionnaire. If the patient did not know English or was illiterate, the questions were asked and filled by the researcher (ASG).

\section{Questionnaire}

The questionnaire was composed of 10 questions asking about knowledge of HPV. Question 1 asked whether the patient has heard about HPV or not. Questions 2-6 asked about the characteristics and transmission knowledge of HPV. Questions 7 and 8 asked whether the patient knew about the role of HPV in causing benign and malignant head and neck lesions. Question 9 asked whether the patient was aware of the way to prevent
HPV infection and question 10 asked whether the patient knew about the role of vaccination in preventing cervical cancers.

\section{Knowledge score}

Knowledge about HPV was assessed using the 10 questions. The respondents answered either yes, no, or don't know. One score was given for a correct answer, zero for a wrong response or don't know answer. The minimum and maximum possible scores were 0 and 10 , respectively. The scores were classified into two levels, either more than or equal to 5 or less than 5 .

\section{Results}

\section{Participant characteristics}

The demographic data of the patients in this study were presented in Table 1. The average age of the participants was 40 years old and the majority of the patients were male. The three most common occupations found in this study were teachers $(16.7 \%)$, housewives $(10.4 \%)$ and farmers $(8.9 \%)$. More than half $(64 \%)$ of the participants were married and most patients graduated from secondary schools $(36.5 \%)$.

\section{Knowledge about HPV}

Knowledge of HPV was derived by assessing the responses to the questionnaire. Table 2 demonstrates the correct answers from each question. Although quite a

Table 1. Demographic Data

\begin{tabular}{|c|c|c|}
\hline Variables & $\begin{array}{l}\text { Number } \\
(\mathrm{N}=192)\end{array}$ & $\%$ \\
\hline \multicolumn{3}{|l|}{ Age (Years) } \\
\hline $16-19$ & 6 & 3.1 \\
\hline $20-29$ & 53 & 27.6 \\
\hline $30-39$ & 61 & 31.8 \\
\hline $40-49$ & 41 & 21.3 \\
\hline $50-59$ & 28 & 14.6 \\
\hline $60-69$ & 3 & 1.6 \\
\hline \multicolumn{3}{|l|}{ Gender } \\
\hline Male & 120 & 62.5 \\
\hline Female & 72 & 37.5 \\
\hline \multicolumn{3}{|l|}{ Occupation } \\
\hline Nurse & 10 & 5.2 \\
\hline Farmer & 17 & 8.9 \\
\hline Driver & 15 & 7.8 \\
\hline Teacher & 32 & 16.7 \\
\hline Shopkeeper & 5 & 7.8 \\
\hline Engineer & 6 & 3.1 \\
\hline Doctor & 9 & 4.7 \\
\hline Businessman & 14 & 7.3 \\
\hline House wife & 20 & 10.4 \\
\hline Student & 15 & 7.8 \\
\hline Others & 39 & 20.3 \\
\hline \multicolumn{3}{|l|}{ Marital status } \\
\hline Married & 123 & 64.1 \\
\hline Unmarried & 69 & 35.9 \\
\hline \multicolumn{3}{|l|}{ Educational attainment } \\
\hline Illiterate & 47 & 24.5 \\
\hline Graduated primary school & 11 & 5.7 \\
\hline Graduated secondary school & 70 & 36.5 \\
\hline Graduated bachelor degree & 64 & 33.3 \\
\hline
\end{tabular}


Table 2. Knowledge about HPV

\begin{tabular}{|c|c|c|c|}
\hline \multirow{2}{*}{$\begin{array}{l}\text { Statement } \\
\text { (correct answer) }\end{array}$} & \multicolumn{3}{|c|}{ Number $(\%)$ of patients who answered } \\
\hline & Yes $(\%)$ & No $(\%)$ & Don't know (\%) \\
\hline 1. Heard about HPV (Yes) & $112(58.4)$ & $69(35.9)$ & $11(5.7)$ \\
\hline 2. HPV infection is more common in young age groups (Yes) & $58(30.2)$ & $37(19.3$ & $97(50.5)$ \\
\hline 3. Female is affected more than male with HPV infection (No) & $59(30.7)$ & $26(13.6)$ & $107(55.7)$ \\
\hline 4. HPV can be transmitted sexually (Yes) & $02(53.1)$ & $4(2.1)$ & $86(44.8)$ \\
\hline 5. HPV can be transmitted by skin to skin contact (Yes) & $54(28.1)$ & $34(17.7)$ & $104(54.2)$ \\
\hline 6. HPV can be transmitted from infected mother to baby (Yes) & $48(25.0)$ & $30(15.6)$ & $114(59.4)$ \\
\hline 7. HPV can cause precancerous and cancerous lesion in head and neck (Yes) & $55(28.6)$ & $23(12.0)$ & $114(59.4)$ \\
\hline 8. HPV can cause oral common warts and oral papillomas (Yes) & $68(35.4)$ & 17 (8.9) & $107(55.7)$ \\
\hline 9. Primary prevention can decrease the risk of HPV infection (Yes) & $56(29.2)$ & $26(13.5)$ & $110(57.3)$ \\
\hline 10. Vaccination plays important role in HPV prevention (Yes) & $36(18.8)$ & $27(14.1)$ & $129(67.1)$ \\
\hline
\end{tabular}

\section{Table 3. Distribution of Knowledge Scores}

\begin{tabular}{lc}
\hline Knowledge level & Number of patients (\%) \\
\hline Answered correctly $\geq 5$ questions & $70(36.5)$ \\
Answered correctly $<5$ questions & $122(63.5)$ \\
\hline
\end{tabular}

number of the participants have heard about HPV, more than $50 \%$ of the patients did not have the basic knowledge about HPV. For example, they did not know that males can be affected more than females. In addition, more than half of the participants had no knowledge regarding the mode of transmission of HPV. Even though more than half of the patients $(53.1 \%)$ were aware that HPV can be transmitted sexually, these participants did not know that HPV can be transmitted via skin to skin contact and from infected mother to baby.

Regarding the knowledge about the association of HPV and head and neck benign and malignant lesions, more than half $(59.4 \%)$ did not know that HPV can cause precancerous and cancerous oral lesions of the head and neck. Furthermore, more than half of the patients did not know that primary prevention can decrease the risk of HPV infection and that vaccination plays an important role in HPV prevention.

Knowledge score about HPV was derived from the correct answers to the 10 questions. Correct answer for each question received one mark while wrong or don't know answers received no mark. The minimum and maximum possible marks were 0 and 10 respectively. The marks were classified into two levels, either more than or equal to 5 and less than 5 . Only $36.5 \%$ of the participants had scores equal to or more than 5 and more than half $(63.5 \%)$ of the patients had scores less than 5 marks (Table $3)$. This result demonstrated lack of knowledge about HPV, role of HPV in causing head and neck benign and malignant lesions, prevention of HPV and role of HPV vaccine in this group of participants.

\section{Discussion}

Many reports mentioned about the knowledge of HPV and cervical malignancy but only a few reports assessed the knowledge of HPV and its association with head and neck benign and malignant lesions. In this study, we conducted a questionnaire survey to investigate the knowledge of Pakistani patients who attended the Dental Department of the Sandeman provincial hospital.
Although more than half of the participants have heard about HPV, a lot of them did not know its basic characteristics and the contribution of this virus in causing head and neck benign and malignant lesions. Comparable to another study which focused on Hispanic women in the U.S., HPV awareness and knowledge, using data from, first, the 2007 health interview national trends survey (HINTS) and second, a cross-section of callers to the national cancer institute's (NCI) cancer information service (CIS) were assessed (Kobetz et al., 2010). The data in the U.S. revealed that HPV awareness was high in both samples $(69.5 \%$ and $63.8 \%$ have heard of the virus) which is similar to our study which indicated that $58.4 \%$ of the patients have heard about HPV. A survey study conducted in Florida, U.S., which composed of 124 students from two southeastern universities showed that $78 \%$ of the students have heard of HPV (Gerend and Magloire, 2008). Interestingly, a study conducted to investigate men's beliefs about HPV-related disease in 608 homosexual, bisexual and heterosexual men revealed that most men $(70.5 \%)$ reported having heard of HPV prior to the internet-based survey (Brewer et al., 2010). More gay and bisexual men have heard of the virus than heterosexual men (79\% vs 62\%). Contrary to our study, a research conducted as a questionnaire survey of 630 students aged between 17-24 years old in Kolkata, India showed that only $15 \%$ of the students have heard the word HPV (Saha et al., 2010). Low level of recognition of HPV was also observed in a study in the U.K. Among 1,032 women attending a well woman clinic in London, it was indicated that only $30 \%$ (316 of 1032) of the participants have heard of HPV and among these women who have heard of HPV, the knowledge about HPV was generally poor (Waller et al., 2003). Non-smokers and those with a history of candida, genital warts, or an abnormal smear result were more likely to have heard of HPV. Awareness of HPV was higher in older (47\%) than younger women $(25 \%)$. These women were white, well-educated and in full-time employment. The data indicated that awareness about HPV may vary according to ethnicity, levels of education, age and previous experience of having sexually transmitted disease.

When knowledge about the mode of transmission of HPV was assessed, 53.1\% of this group of Pakistani patients perceived that HPV can be transmitted sexually. However, more than half of them did not know that HPV can be transmitted through skin to skin contact and from 
infected mother to baby. Compared to the data from the CIS and the HINTS samples, it was revealed that, from the two groups, $66.8 \%$ and $70.4 \%$ perceived that HPV can be transmitted sexually, respectively (Kobetz et al., 2010). In a study conducted with 300 women from three ambulatory obstetrics and gynecology practices in Philadelphia, U.S., it was revealed that the knowledge about the mode of transmission of HPV was reported to be different in two age groups. In the younger age group (19-26 years old, $\mathrm{n}=131), 77.9 \%$ thought that HPV can be transmitted sexually compared to $44.3 \%$ in the older age group (4070 years old, $n=149$ ) (Montgomery and Smith-Glasgow, 2012). A study conducted to investigate the knowledge about sexually transmitted infections among university students in Sao Paulo, Brazil, reported that $68.8 \%$ and $76.1 \%$ of male and female students perceived that HPV infection is sexually transmitted (Caetano et al., 2010). In contrast, only 205 of 1,032 (19.9\%) women attending a well woman clinic in London, U.K. perceived that HPV can be sexually transmitted (Waller et al., 2003). The data implied that different groups of participants may have different levels of knowledge and education regarding the mode of transmission of HPV.

Knowledge about the contribution of this virus in causing benign and malignant diseases was also assessed in this study. The majority of the previous studies assessed the knowledge of HPV in causing genital benign and malignant lesions. In a study conducted among the U.S. Hispanic women, it was revealed that the CIS sample, which was more impoverished and less acculturated than their HINTS counterparts, were less able to correctly identify that HPV causes cervical cancer (67.1\% vs 78.7\%) (Kobetz et al., 2010). Nevertheless, the percentage of participants who perceived that HPV can cause cervical cancer was very high in both groups. In another study conducted in Philadelphia between two women age groups, $80.2 \%$ of women in the younger age group thought that cervical cancer and precancerous cells are associated with the presence of HPV whereas only $26.8 \%$ of women in the older age group perceived this information (Montgomery and Smith-Glasgow, 2012). A very high percentage of students $(92 \%)$ who correctly identified the causal link between HPV and cervical cancer was observed in a study conducted in Florida (Gerend and Magloire, 2008). In contrast, low levels of knowledge regarding the contribution of HPV in inducing genital benign and malignant lesions were observed in some studies. In a study conducted in London, only $12.3 \%$ (127 out of 1,032$)$ of women attending a well woman clinic recognized that HPV is a cause of cervical cancer (Waller et al., 2003). In Sweden, an investigation of 572 high school students from five different high schools revealed lack of knowledge about HPV and its association with cervical cancer (Hoglund et al., 2009). Only 5.4\% of the students have heard about HPV and only $1.2 \%$ recognized that HPV can cause cervical cancer (Hoglund et al., 2009). In a study conducted with female students of premier colleges in Kolkata, India, only $4 \%$ of the students recognized that cervical cancer is caused by an infection (Saha et al., 2010). In a study conducted with 608 gay or bisexual or heterosexual men in North Carolina, U.S., it was found that only $41 \%$ and $24 \%$ of them knew that HPV can cause genital warts and anal cancers, respectively (Brewer et al., 2010). Most men believed that sexual behavior causes genital warts $(70 \%)$ and anal cancer $(54 \%)$. Interestingly, the majority of men believed that tobacco use is the main cause of oral cancer (89\%) and only $23 \%$ of these men recognized HPV as a causative factors of oral cancers. In comparison to our data, only $28.6 \%$ of our Pakistani participants recognized that HPV can cause precancerous and cancerous lesions in head and neck regions and $35.4 \%$ of them thought that HPV can also cause common oral warts and papillomas. More than half of the patients did not have any knowledge about this.

Prevention of HPV infection can be achieved through several ways. Examples are consistent use of condom, abstinence from having multiple sex partners, being monogamous and receiving HPV vaccine. In this study, more than half of the participants $(57.3 \%)$ were not sure whether primary prevention could prevent HPV infection. Lack of knowledge on how to prevent HPV infection was found in some studies. For example, in a study, awareness of HPV among women attending a well woman clinic in the U.K. was assessed (Waller et al., 2003). Although half of the patients who have heard about HPV knew that contraceptives could not protect HPV transmission, $36.8 \%$ of these participants were not sure about this (Waller et al., 2003). In contrast, some studies showed good knowledge about HPV prevention among the participants. For instance, in one study which assessed awareness and knowledge about HPV in a group of young adults in Florida, $84 \%$ of the patients perceived that a person's chance of getting HPV increases with the number of sexual partners (Gerend and Magloire, 2008). Interestingly, one study reported that misconceptions and misunderstandings as well as lack of knowledge about HPV prevention could be addressed if education was given to the target groups. When a brief information regarding HPV and oral sex was given to a group of young adults, it was found that participants who read the information about HPV and oral sex and cancer reported greater knowledge about perceived risks and lower their willingness to engage in oral sex compared to those who did not read the information (Stock et al., 2012). The data indicated that in some groups of participants, knowledge about the prevention of HPV was poor. However, if adequate information on how to prevent HPV infection is given to these people, the knowledge about the prevention of HPV transmission will be improved which can help prevent HPV infection.

HPV prevention by vaccination programs have been started in many parts of the world. Commercial vaccines currently available are quadrivalent Gardasil ${ }^{\circledR}$ which prevents infection with HPV types 16, 18, 6 and 11 and bivalent Cervarix ${ }^{\circledR}$ which prevents HPV types 16 and 18 infection (Prabhu and Wilson, 2013). These vaccination programs were aimed at the genital, HPV-related lesions and were proven to be a successful policy (Wierzbicka et al., 2014). Recommendation is aimed at preventing HPV and associated cervical and other anogenital cancers with the routine use of HPV vaccines for males and females. Therefore, it should be interesting to know 
whether our group of patients had any knowledge about HPV vaccination. It was found that most participants in our study $(67.1 \%)$ did not know whether vaccination plays an important role in HPV prevention. Similar to our study, knowledge about HPV vaccine was limited for some groups of participants. For instance, a study conducted with Swedish high school students revealed that the knowledge about vaccine was limited although their attitude to condom use when having sex with a new partner was positive (Hoglund et al., 2009). In a study in India, although awareness and knowledge of cervical cancer among female students of premier colleges in Kolkata was poor, $75 \%$ of the students would like to have protective vaccination (Saha et al., 2010). Although HPV vaccination may help prevent HPV infection, some concerns have been raised (Hoglund et al., 2009). Since these vaccines can prevent only some types of HPV infection, patients who receive these vaccines are still prone to other types of HPV infection. Moreover, if the participants are more aware of the association between HPV and cervical cancer, it might lead to an increased use of condoms or having fewer partners. However, an intention to use a condom or having pap smear routinely or abstinence from having multiple sex partners may decrease if they were vaccinated. In addition, there is a potential risk that vaccination against one sexually transmitted disease (HPV) can give young women and men a sense of false security and lead to increased sexual risk-taking which in the long term could increase the spread of other sexually transmitted diseases (Hoglund et al., 2009).

It is indicated that the knowledge of our patients was poor since more that half $(63.5 \%)$ of the participants had score less than 5. However, some studies suggested that intervention by giving correct information about HPV infection could help improve the knowledge of the participants (Daley et al., 2011). Since oral healthcare providers have several important roles in the prevention of HPV-associated benign and malignant lesions, these providers should have correct information about HPV to be able to convey this information to their patients. Additionally, they must be able to recognize HPV-related oral lesions and screen these HPV-associated benign and malignant oral lesions that may emerge in the oral cavity and surrounding tissue such as the oropharynx and tonsillar areas so that they can refer patients for proper management.

In conclusion, it was shown that the participants in our study had low level of knowledge regarding the contribution, transmission and prevention of HPVassociated oral diseases. Adequate knowledge about HPV should be provided to Pakistani patients to be able to prevent the occurrence of HPV-associated benign and malignant lesions of the head and neck areas.

\section{Acknowledgements}

This project was supported by Mahidol University research grant, Thailand and in part by the office of the higher education commission and Mahidol university, Thailand under the national research universities initiative. All the authors have no conflict of interest to declare.

\section{References}

Brewer NT, Ng TW, McRee AL, Reiter PL (2010). Men's beliefs about HPV-related disease. J Behav Med, 33, 274-81.

Caetano ME, Linhares IM, Pinotti JA, et al (2010). Sexual behavior and knowledge of sexually transmitted infections among university students in Sao Paulo, Brazil. Int $J$ Gynaecol Obstet, 110, 43-6.

Carr J, Gyorfi T (2000). Human papillomavirus. Epidemiology, transmission and pathogenesis. Clin Lab Med, 20, 235-55.

Chang F, Syrjanen S, Kellokoski J, Syrjanen K (1991). Human papillomavirus (HPV) infections and their associations with oral disease. J Oral Pathol Med, 20, 305-17.

Daley E, DeBate R, Dodd V, et al (2011). Exploring awareness, attitudes and perceived role among oral health providers regarding HPV-related oral cancers. J Public Health Dent, 71, 136-42.

Gerend MA, Magloire ZF (2008). Awareness, knowledge and beliefs about human papillomavirus in a racially diverse sample of young adults. J Adolesc Health, 42, 237-42.

Gichki AS, Buajeeb , Doungudomdacha S, Khovidhunkit SP (2012). Detection of human papillomavirus in normal oral cavity in a group of Pakistani subjects using real-time PCR. Asian Pac J Cancer Prev, 13, 2299-304.

Hoglund AT, Tyden T, Hannerfors AK, Larsson M (2009). Knowledge of human papillomavirus and attitudes to vaccination among Swedish high school students. Int J STD AIDS, 20, 102-7.

Kobetz E, Kornfeld J, Vanderpool RC, et al (2010). Knowledge of HPV among United States Hispanic women: opportunities and challenges for cancer prevention. J Health Commun, 3, 22-9.

Kojima A, Maeda H, Kurahashi N, et al (2003). Human papillomaviruses in the normal oral cavity of children in Japan. Oral Oncol, 39, 821-8.

Kulasingam SL, Hughes JP, Kiviat NB, et al (2002). Evaluation of human papillomavirus testing in primary screening for cervical abnormalities: comparison of sensitivity, specificity and frequency of referral. Jama, 288, 1749-57.

Montgomery K, Smith-Glasgow ME (2012). Human papillomavirus and cervical cancer knowledge, health beliefs and preventive practices in 2 age cohorts: a comparison study. Gend Med, 9, 55-66.

Prabhu SR, Wilson DF (2013). Human papillomavirus and oral disease-emerging evidence: a review. Aust Dent J, 58, 2-10.

Reiter PL, Brewer NT, Smith JS (2010). Human papillomavirus knowledge and vaccine acceptability among a national sample of heterosexual men. Sex Transm Infect, 86, 241-6.

Saha A, Chaudhury AN, Bhowmik P, Chatterjee R (2010). Awareness of cervical cancer among female students of premier colleges in Kolkata, India. Asian Pac J Cancer Prev, 11, 1085-90.

Stock ML, Peterson LM, Houlihan AE, Walsh LA (2012). Influence of oral sex and oral cancer information on young adults' oral sexual-risk cognitions and likelihood of HPV vaccination. J Sex Res, 50, 95-102.

Waller J, McCaffery K, Forrest S, et al (2003). Awareness of human papillomavirus among women attending a well woman clinic. Sex Transm Infect, 79, 320-2.

Wierzbicka M, Jozefiak A, Jackowska J, Szydlowski J, Gozdzicka-Jozefiak (2014). A HPV vaccination in head and neck HPV-related pathologies. Otolaryngol Pol, 68, 157-73. 